\title{
Multi-gram Mechanosynthesis of Salophen-Complex: A Comparative Analysis
}

\author{
Varun Kumar Singh, ${ }^{\dagger}$ Alex Chamberlain-Clay ${ }^{\dagger}$, How Chee Ong, Felix León, Gavin Hum, Mian Yang \\ Par, Patrick Daley-Dee, Felipe García*
}

School of Physical and Mathematical Sciences, Division of Chemistry and Biological Chemistry, Nanyang Technological University, 21 Nanyang Link, 637371, Singapore

KEYWORDS: Mechanosynthesis, multi-gram, group 13, salen, salophen, E-factor, sustainable

\begin{abstract}
Mechanosynthesis is a powerful alternative to traditional solvent-based synthesis as it is environmentally benign, allows for higher yields in shorter reaction times, while minimizing the use of solvents. Although mechanochemical routes are becoming increasingly mainstream in synthetic laboratories, up-scaled examples for main group complexes are still rare. Here, motivated by the practical implementation of mechanosynthesis, we demonstrated the synthesis of salen and salophen complexes. The herein reported synthesis displays low E-factor and process mass intensity compared to conventional solution methods. In addition, analyses evaluating environmental parameters, energy consumption and production cost have been performed, showing the multiple advantages mechanochemistry has over conventional solutionbased synthesis.
\end{abstract}

\section{Introduction}

Mechanosynthesis is an attractive alternative to conventional solution-based synthesis, since it not only addresses several environmental concerns associated with the use of large volume of solvents but also often offers shorter reaction times and higher yields. ${ }^{1,2}$ Uniquely, mechanochemistry also offers opportunities to prepare chemical compounds that are otherwise difficult or impossible to prepare by solution methods owing to limited solubility of the starting material and the limited stability of the product in solution., ${ }^{3,4}$ In recent years, mechanochemistry has increasingly been applied as a benign synthetic route in a wide range of fields.5-11 (e.g., organometallic complexes, ${ }^{12-14}$ organo-catalysts, ${ }^{15}$ cocrystals $^{16-18}$, metal-organic frameworks, ${ }^{19-21}$ alloys, ${ }^{22}$ composites, ${ }^{23}$ etc.)

Among organometallic species, salen and salophen complexes have received considerable attention in stereoselective synthesis ${ }^{25}$, photoredox catalysis ${ }^{26}$, polymer synthesis, ${ }^{27-30}$ chemical sensors ${ }^{31,32}$ and OLEDs. ${ }^{33,34}$ However, their typical synthetic procedures generally require the use of hazardous organic solvents at elevated temperatures ${ }^{35-39}$. For instance, Fulton and co-workers ${ }^{40}$ reported the synthesis of a saturated "salen type" monometallic and bimetallic aluminium complexes of piperazine-based ligands (Figure 1a). In their synthesis, $c a$. $32 \mathrm{~g}$ of ligand required $200 \mathrm{~mL}$ of methanol under reflux conditions, with an additional $500 \mathrm{~mL}$ of diethyl ether for workup. This methodology results in high E-factors ${ }^{41-45,49}$ (i.e., total weight of all the solvents used in this synthesis divided by the weight of isolated product). This severely impacts the practical industrial implementation of such complexes, since large scale synthesis leaves a large environmental footprint

In this context, mechanochemical processes coupling 1,2diamine and salicylaldehyde is a promising approach for the synthesis of salen or salophen ligands and their corresponding complexes in an environmentally benign manner.

In

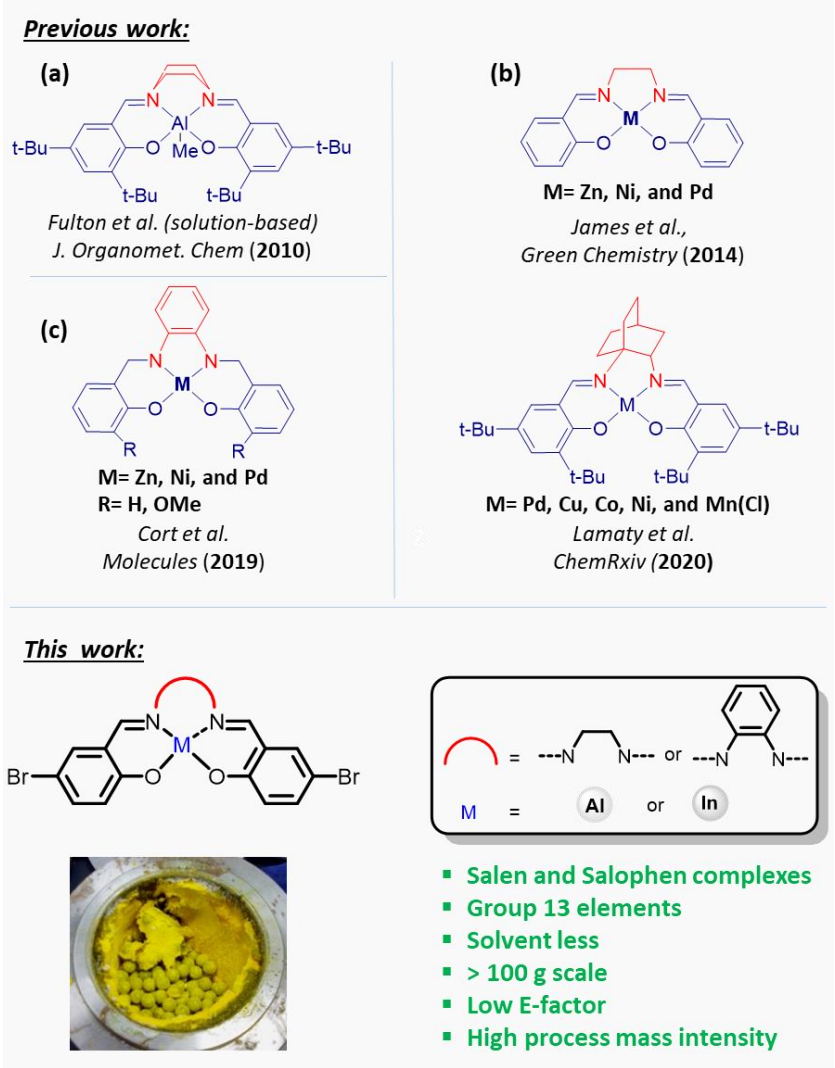

Figure 1. Top: Previous studies on (a) large-scale solutionbased synthesis of group 13 salen type species, mechanochemical synthesis of (b) salen and (c) salophen species.

fact, the mechanosynthesis of these species have been performed at laboratory-scale and has been recently reported. However, no large-scale synthesis has been previously reported. 
(a)

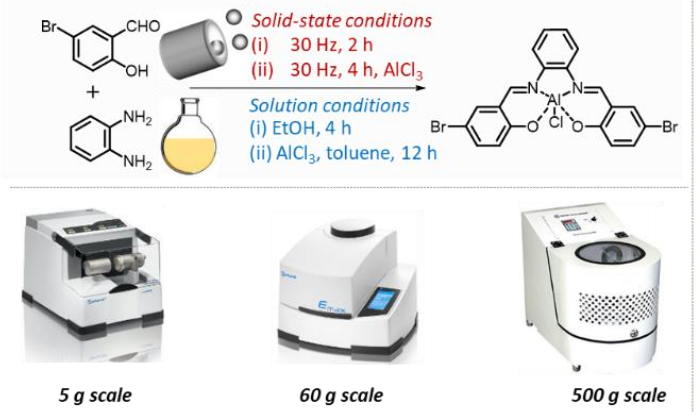

(b)

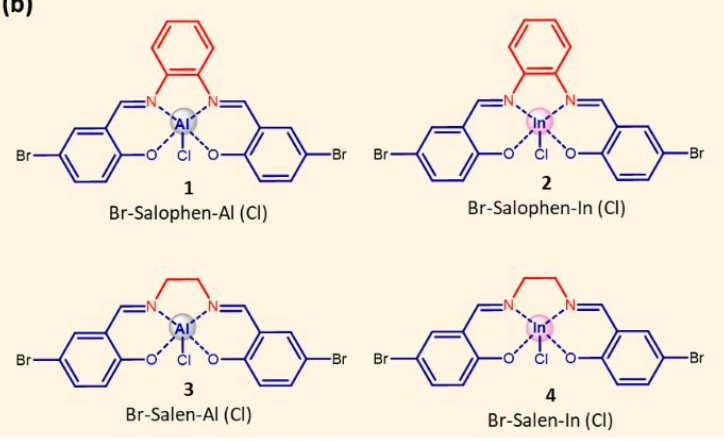

Figure 2. (a) Reaction conditions for both mechanochemical and conventional-solution synthetic routes (top); images of ball milling equipment used throughout this work - vibratory ball-mill (VBM), a one station planetary ball mill (PBM), and a four station PBM (from left to right respectively). (b) Proposed molecular structure for the reported salen and salophen complexes.

Recently, the James group ${ }^{15}$ has shown mechanosynthesis of salen complexes with transition metals (Figure 1b, top) under liquid assisted grinding (LAG) conditions (i.e., a minimal amount of solvent is used). In addition, the one-pot up-scaled synthesis of a zinc salen complex ( $c a .4 \mathrm{~g}$ ) was also demonstrated. While the described method opened opportunities for the mechanochemical synthesis of these species, the reported synthesis still depends on the use of solvent additives to accelerate the reaction. However, at industrial scales, the amount of solvent required would be vastly increased. In fact, $80 \%$ of the energy used in the separation processes globally is associated with distillation and evaporation methods. ${ }^{46}$ Therefore, the need for solvent removal - which generally is an energy-intensive process remained inevitable. Later, Cort and co-workers ${ }^{47}$ reported the LAG mechanosynthesis of a series of salophen transition metals complexes (i.e., $\mathrm{M}=\mathrm{Zn}, \mathrm{Ni}, \mathrm{Pd}$ ) - See Figure 1c. This method proceeded with yields ranging from 60 to $68 \%$ for the complexes reported. Moreover, complexation with chloride salts also remained unsuccessful throughout all their syntheses. More recently, the Lamaty group also reported mechanosynthesis of manganese salen complexes based on 1,2-bicyclo[2.2.2] octane bridge ( $\mathrm{M}=\mathrm{Pd}, \mathrm{Co}, \mathrm{Co}, \mathrm{Ni}$ and $\mathrm{Mn}(\mathrm{Cl})$ ). These species, synthesized in the presence of sodium chloride as a salt additive, were produced in moderate to good yields (i.e., 62 to $98 \%$ ) in the milligram scale (i.e.,, 9.8 to $129 \mathrm{mg}$ ). ${ }^{48}$

Even though mechanosynthesis has demonstrated to have the potential to be a disruptive technology in industry, there are several aspects, however, that hinder its broader adoption. These issues include (i) the lack of comparable performance data between different synthetic methodologies, (ii) the impact of large scale production on environmental factors (e.g., E-factors), and (iii) the lack the need for new experimental designs at industrially relevant scale, among others. Furthermore, harmful and hazardous by-products generated during reaction no longer have a solvent system to capture or dilute them. This could potentially pose a serious handling and safety issues for solvent less techniques at industrial scales, versus their solvent-based counterparts. Hence, the cross examination of large scale protocols across different reaction media (i.e., mechanochemical vs solution-based) is necessary to produce environmental footprint data required for effective industrial implementation of mechanochemical synthesis.
Since a practical implementation of the large-scale mechanochemical synthesis of metal complexes would largely depend on its environmental footprint (i.e., green metrics), we have investigated a series of key environmental parameters - namely, (i) E-factor, (ii) process mass intensity (PMI), (iii) reaction mass efficiency (RME), (iv) atom economy (AE), and ( $v$ ) energy efficiency and compared the values obtained with conventional solution-based synthesis. The main goal of this report is not only to enhance the knowledge (from a practical perspective) necessary to assess the scalability processes in mechanosynthesis but also to help provide quantifiable metrics to compare with solution-based methodologies.

Herein we report the solvent-free mechanochemical synthesis of bromo-salen and bromo-salophen ligands and their group 13 complexes. In addition, as a model, we have determined the E-factors for a multi-gram synthesis of compound $\mathbf{1}$ and compared it with conventional solutionbased methods at different scales, up to three orders of magnitude (see Fig. 2a). Overall, mechanosynthesis exhibited very low E-factors, low energy consumption and shorter reaction times at room temperature.

\section{Results and Discussion}

Schiff base complexes containing bromine atoms have shown to possess promising anti-cancer and -proliferative bioactivity. Moreover, they can be used as parent compound for synthesis of a wide range of species via Suzuki and Sonogashira coupling reactions. Hence, these chemically versatile species were used as a model system throughout our study.

Previously reported synthesis of salen and salophen ligands and complexes either used LAG during the ligand synthesis or metal complexation steps (in the case of transition metals), or at least one of the reactants is liquid (i.e., reactant acting as LAG). To the best of our knowledge, there are no reports on the neat mechanochemical synthesis of salen and salophen ligands and their group 13 complexes (LAG was required for full conversion) or a comprehensive scaling-up studies (Fig.2b). Inspired by concepts put forth by Ito et al., ${ }^{49,50}$ we decided to expand our model to cover both solid-liquid and solid-solid type synthesis. 
We started our studies with the synthesis of salen (solidliquid) and salophen (solid-solid) ligands. These species are traditionally obtained from the condensation of salicylaldehydes and diamines ${ }^{51}$ under reflux in protic solvents (e.g., methanol, ethanol, etc.)..$^{52}$ Alternatively, these species can be produced mechanochemically via neat or LAG conditions. $1,15,47,53$

In a typical reaction, 5-bromosalicylaldehyde and a diamine (ethylenediamine, and 1, 2-phenylenediamine for salen and salophen ligands, respectively) were ground together in a $15 \mathrm{ml}$ milling jar with a $8 \mathrm{~mm}$ diameter ball (both stainless steel) - see Fig. 3a). Samples were taken every 30 minutes and analysed by ex situ ${ }^{1} \mathrm{H}$ NMR spectroscopy to monitor the reaction. The mechanochemical synthesis of the ligands proceeded smoothly and achieved full conversion after 2 hours. Since the reaction produced water as by-product, the solids obtained were oven-dried to afford free flowing yellow powders. The ${ }^{1} \mathrm{H}$ NMR spectra displays broad singlet peaks at 13.12 and $12.92 \mathrm{ppm}$, and 8.28 and $8.928 .15 \mathrm{ppm}$ for phenolic and iminic protons respectively, which is in line with the previous reports for analogous $\mathrm{Br}$-salen and $\mathrm{Br}$ salophen ligands, respectively (See Supporting Information, SI). ${ }^{15}$ Both ligands, Br-salen and Br-salophen, were obtained in high yields (i.e., 92\% and 98\%, respectively) - see Fig. $\mathbf{3 b}$.

After the successful synthesis of the targeted salen and salophen ligands, they were then treated with $\mathrm{AlCl}_{3}$ or $\mathrm{InCl}_{3}$ mechanochemically to afford a total of four complexes: $\mathrm{Br}$ salophen-Al (Cl) (1); Br-salophen-In (Cl) (2); Br-salen-Al (Cl) (3) and Br-salen-In (Cl) (4). The complexation reaction was confirmed by ${ }^{1} \mathrm{H}$ NMR spectroscopy by the absence of phenolic protons and the downfield shift for both iminic protons as compared to signals in free salen or salophen ligand (recorded in $\mathrm{CDCl} 3$ and DMSO, respectively)- see SI.

Aluminium complexes with both salen and salophen ligands were obtained in higher yields than their indium counterparts. For instance, the Br-salophen aluminium complex (compound 1) achieved full conversion $(97 \%$ yield) after 4 hours of reaction time, whereas Br-salophenIn (compound 2) was obtained in a lower yield ( $70 \%$ yields) under the same conditions. We attribute this to the difference in coordination environment between salen and salophen ligands, as the salophen ligand displays a more rigid planar structure which will better accommodate the smaller aluminium atom.

Notably, the aluminium complexes exhibited higher stability to air and moisture over a period of three months, in contrast with their indium counterparts, which were hydrolysed into the aldehyde/diamine over the same period. Hence, to prevent hydrolysis, magnesium sulfate was used as dehydrating agent during the synthesis of complex 2, 3 and $4-$ which achieved full conversion after 5 hours of milling.

\section{Assessment of environmental impact.}

Despite being regarded as environmentally benign and sustainable, modern examples of scaled-up mechanochemical synthesis as well as detailed assessments

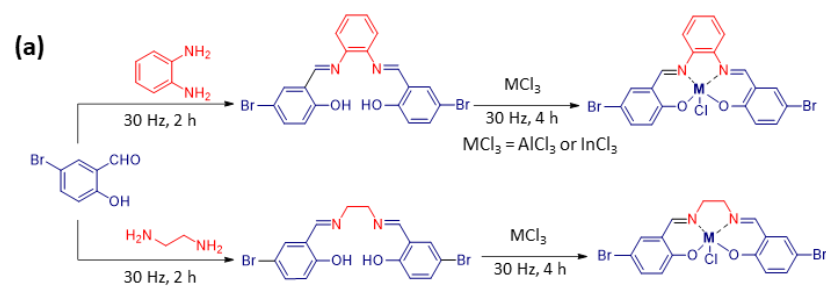

(b)

\begin{tabular}{|cccccc|}
\hline Entry & Compound & $\begin{array}{c}\text { Frequency } \\
(\mathbf{H z})\end{array}$ & Time (h) & Conversion (\%) & Yield (\%) \\
\hline 1 & Br-Salen & 30 & 2 & 100 & 92 \\
2 & Br-Salophen & 30 & 2 & 100 & 98 \\
3 & Br-Salen-Al (1) & 30 & 4 & 100 & 77 \\
4 & Br-Salophen-Al (2) & 30 & 4 & 100 & 97 \\
5 & Br-Salen-In (3) & 30 & 4 & 100 & 70 \\
6 & Br-Salophen-In (4) & 30 & 4 & 100 & 70 \\
\hline
\end{tabular}

Figure 3. (a) Synthesis route for group 13 salen and salophen complexes under mechanochemical conditions (b) Reaction conditions and results of mechanosynthesis of salen and salophen complexes.

and comparison of its environmental impacts with respect we contrasted the synthesis of complex $\mathbf{1}$ in both mechanochemical and solution-based methods. to conventional syntheses in the scientific literature remains rare. Using conventional solution-based synthesis as a case study, we contrasted the synthesis of complex 1 in both mechanochemical and solution-based methods.

Specifically, we performed a detailed assessment of the environmental impact of large scale mechanosynthesis of $\mathrm{Br}$-Salophen- $\mathrm{Al}(\mathrm{Cl})$ (i.e., complex 1) and compared it with the corresponding conventional solution-based synthetic route. Specifically, different scale batches viz., $0.1 \mathrm{~g}, 1 \mathrm{~g}, 30$ $\mathrm{g}, 60 \mathrm{~g}, 120 \mathrm{~g}$ were prepared using both methods and their green metrics were assessed. The synthesis of small-scale reactions (i.e., $0.1 \mathrm{~g}$ and $1 \mathrm{~g}$ ) were performed in a VBM using one $8 \mathrm{~mm}$ steel ball. For larger scales (i.e., $60 \mathrm{~g}$ and $120 \mathrm{~g}$ ) a PBM was used, with $500 \mathrm{~g}$ of steel balls per $60 \mathrm{~g}$ of reagents to maintain the ball to reagent ratio to $\sim 9: 1$ for all syntheses.

Planetary mills are ideal for scale-up and they work based on the principle of planetary motion as the jar rotates around a central axis to create centrifugal forces, thus emulating the effect of gravity in industrial scale roller mills. ${ }^{54-56}$ Theoretically, although each jar has a total capacity of $125 \mathrm{~g}$, each jar was loaded up to $60 \mathrm{~g}$ of reagents in this study (two jars were used for the $120 \mathrm{~g}$ scale reaction). Even at this large scale, we achieved full conversion with $95 \%$ yield as analysed by ${ }^{1} \mathrm{H}$ NMR spectroscopy (Fig. 3b). For comparative purposes, the synthesis of $\mathbf{1}$ by conventional solution-based methods in ethanol and toluene were performed, which produced comparable overall yields (94\%).

When the reactions were scaled up, the large amount of $\mathrm{HCl}$ gas resulted in two technical issues. Firstly, the gases released resulted in jar corrosion with continuous usage. 


\begin{tabular}{|c|c|c|c|c|c|}
\hline Entry & Method & Yield (\%) & E-factor ${ }^{\mathbf{a}}$ & $\mathbf{P M I}^{\mathbf{b}}$ & RME (\%) \\
\hline \multicolumn{6}{|c|}{ Mechanosynthesis } \\
\hline 1 & $0.1 \mathrm{~g}$ & 88 & 0.49 & 0.48 & 67 \\
\hline 2 & $1 \mathrm{~g}$ & 97 & 0.30 & 1.30 & 76 \\
\hline 3 & $30 \mathrm{~g}$ & 69 & $0.70(3.16)$ & $1.70(21.51)$ & 58 \\
\hline 4 & $60 \mathrm{~g}$ & 82 & $0.49(2.54)$ & $1.49(17.76)$ & 67 \\
\hline 5 & $120 \mathrm{~g}$ & 95 & $0.65(2.99)$ & 1.65 (20.98) & 60 \\
\hline \multicolumn{6}{|c|}{ Solution synthesis } \\
\hline 6 & $0.1 \mathrm{~g}$ & 56 & 4.93 & 41 & 49 \\
\hline 7 & $1 \mathrm{~g}$ & 85 & 2.60 & 20.91 & 61 \\
\hline 8 & $30 \mathrm{~g}$ & 66 & $4.17(6.67)$ & $31.52(51.41)$ & 45 \\
\hline 9 & $60 \mathrm{~g}$ & 86 & $2.27(4.09)$ & $20.00(34.34)$ & 70 \\
\hline 10 & $120 \mathrm{~g}$ & 94 & $2.81(4.96)$ & $23.44(40.46)$ & 61 \\
\hline
\end{tabular}

a Values in parenthesis correspond when a base is used to mop out the $\mathrm{HCl}$ formed and water is used to wash the final product (calculations include $90 \%$ solvent recovery). b Values in parenthesis correspond to the use of base and water during the synthesis and purification, respectively.

Secondly, there is a build-up of dangerous amounts of pressurised $\mathrm{HCl}$ gas. Hence, to bypass both issues, triethylamine (TEA) was added to the reaction mixture to act as $\mathrm{HCl}$ scavenger. In this case, the final product was washed with water to remove triethylammonium chloride.

All the quantitative data collected throughout our syntheses at different scales, for both mechanosynthesis and traditional solution-based methods was tabulated and used to estimate the environmental impact of the different synthetic routes used. To estimate the environmental impact, a green chemistry metrics (GCM) ${ }^{57}$ approach was used, and E-factor, ${ }^{58}$ atom economy (AE), process mass intensity (PMI) and reaction mass efficiency (RME) for both the synthetic routes were calculated. Table 1 summarises these metrics for both mechanosynthesis and the solutionbased synthesis. Detailed calculations can be found in the SI (section D). Under mechanochemical conditions, low Efactors were observed, which remained low throughout all the reaction scales, ranging from 0.30 to 0.70 (Table 1 and Figure 4a). In contrast, under conventional solution-based synthetic routes, the E-factors were between $c a$. 5- and 10fold greater (ranging between 2.60 to 4.17). These reactions were performed in the absence of a base, which results in the previously highlighted issues related to $\mathrm{HCl}$ gas being evolved during the reaction.

In the presence of a base (i.e., TEA), the amount of waste produced increased since triethylammonium chloride was formed as by-product - which needed to be removed during the final purification stage. The calculated E-factor values, however, remained low compared to traditional solutionbased methods (i.e., 2.54 to 3.16 vs. 4.09 to 6.67 for mechanochemical and solution routes, respectively). Several factors, however, should be considered when interpreting E-factors in the presence of a base. For instance, the use of a base to trap $\mathrm{HCl}$ gas could be bypassed when specialized milling apparatus designed to vent (or collected for further use) the $\mathrm{HCl}$ gas produced is used. Hence, the additional step for removal of this salt byproduct and the use of required solvent (water in this case) could easily be avoided, which drastically reduce the overall waste generation. RME and AE values for both processes were similar with RME values at $\sim 60 \%$ for $120 \mathrm{~g}$ scale (Figure $4 \mathbf{b}$ ), and the $\mathrm{AE}$ remained at $78 \%$ throughout our studies.

Next, we determined the PMI to see how well it aligns with the E-factor assessment (see Eq. 1). PMI is a green chemistry metric recently developed by the ACS GCI Pharmaceutical Roundtable, ${ }^{59,60}$ This parameter helps determine the sustainability of a process by defining the ratio between the quantity of raw materials involved in the process and the amount of product obtained. This metric, which focuses on the process input rather than output, is an ideal metric to compare between mechanosynthesis and conventional solution method. For this study, we have used PMI calculation equation recently given by Anadraos (Eq. 1). ${ }^{61}$ Whereas $P M I_{1 \rightarrow N}$ refers to overall PMI from step 1 to step $N$ of linear synthesis. $\mathrm{x}_{1}, \mathrm{X}_{2}, \ldots, \mathrm{x}_{\mathrm{n}}$ refers to mass input of raw materials in step $1,2, \ldots, n^{\text {th }}$, respectively (see $\boldsymbol{E q} .2$ ).

$$
\begin{gathered}
\text { PMI }=\frac{\text { Total quantity of starting materials }(\mathrm{kg})}{\text { Quantity of product }(\mathrm{kg})} \\
\underset{1 \rightarrow N}{\mathrm{PMI}}=\frac{x 1+x 2+. .+x N-(m p 1+m p 2+. .+m p N-1)}{\mathrm{mpN}}
\end{gathered}
$$

The terms $m p 1, m p 2, \ldots, m p n$ refers to mass of product of step $1,2, \ldots,(n-1)^{\text {th }}$, respectively, whereas $m p N$ refers to mass of product of final step. The PMI values for different scales were calculated using these equations (i.e., Eq. 1 and Eq. 2) and the data is available in the SI (section D-2).

The mechanochemical synthesis at all the studied scales show lower PMI values $(0.48$ - 1.70) than their solutionbased counterparts (20 - 41) (Figure 4c). Moreover, aligned to what was observed for E-factors, the use of a base (i.e., TEA) increased the PMI values as a result of the extra purification step required (i.e., washing with water). 
a

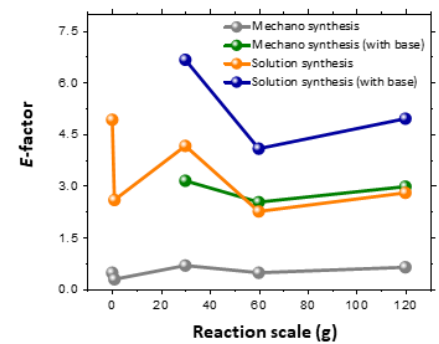

b

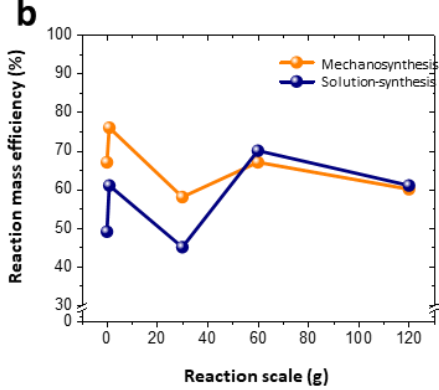

C

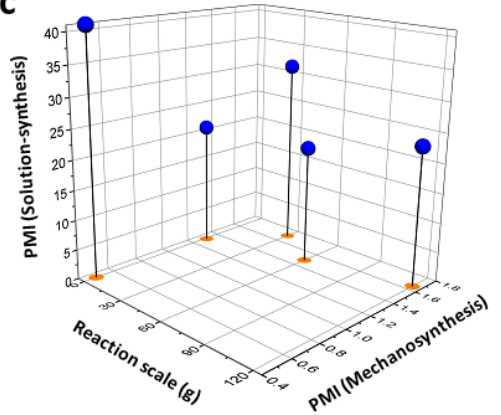

d

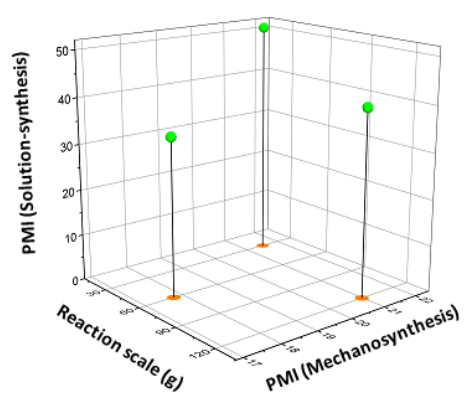

e

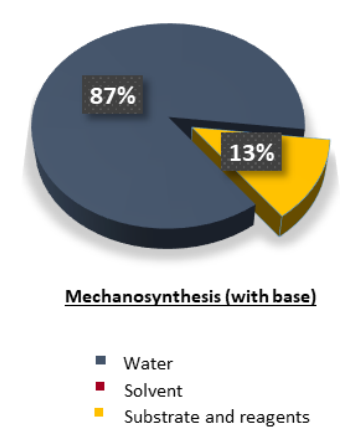

f

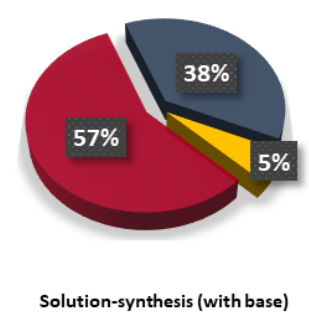

Figure 4. (a) E-factor for mechanosynthesis and solution method of complex Br-salophen- $\mathrm{Al}$ ( $\mathrm{Cl}$ ); the graph shows values with and without the use of base (b) Reaction mass efficiency of mechanosynthesis and solution method (c) Process mass intensity of mechanosynthesis (xy plane; in orange) and solution method (xz plane; in blue); the values are without the use of base in calculations (d) Process mass intensity of mechanosynthesis (xy plane; in orange) and solution method (xz plane; in green); the values are with use of base and is given only for 30, 60 and $120 \mathrm{~g}$ scale (e) Composition of overall PMI of mechanosynthesis (f) Composition of overall PMI of solution method

However, despite using a base and incorporating an additional washing step, the mechanochemical route still displays lower PMI values (i.e., ranging from 17.76 to 21.51 vs. 34.34 to 51.41), which further emphasizes the reduced environmental footprint of mechanosynthesis vs tradition solution-based methods. (Figure 4d).

As previously highlighted, the use of a suitable mechanochemical setup capable of bypassing the use of bases will allow for a low PMI throughout the synthetic process since a major contributor to the increased PMI is due to the use of water during the purification step. However, it should be noted that water has a negligible carbon footprint, and the increased PMI from the aqueous wash does not accurately represent the actual environmental impact as a result.

Finally, we analysed the reaction composition to understand the role of each component in the context of the sustainability of the two synthetic routes studied (Figures 4e and 4f). For the largest scale studied (120 g scale) involving the use of a base (TEA) and water, $87 \%$ of the materials used is water in the mechanochemical route $13 \%$ substrates and reagents). Whereas, for solution synthesis, more than $57 \%$ of the materials used are organic solvents (5\% substrates and reagents). These results further indicate that mechanosynthesis has a lower environmental footprint than conventional solution-based synthesis.

Next, we examined some technical and safety factors during the mechanosynthesis of salen- and salophen complexes, which are associated with the production of $\mathrm{HCl}$ gas throughout the reaction. We have calculated the amount of $\mathrm{HCl}$ produced for the different reaction scales (Figure 5) which will have to be taken into consideration since it influences: (i) the milling media used (corrosion resistant vs not resistant), (ii) the type of jars used (vented $v s$ pressurised), (iii) the reaction composition (presence vs absence of base), and (iv) the appropriate personal protection measures, PPEs and safety protocols to be implemented through a given synthesis. For instance, the use of vented milling jars designed to vent the $\mathrm{HCl}$ produced will not only result in a lower environmental footprint (especially if it can be re-utilised) but also minimise jar corrosion.

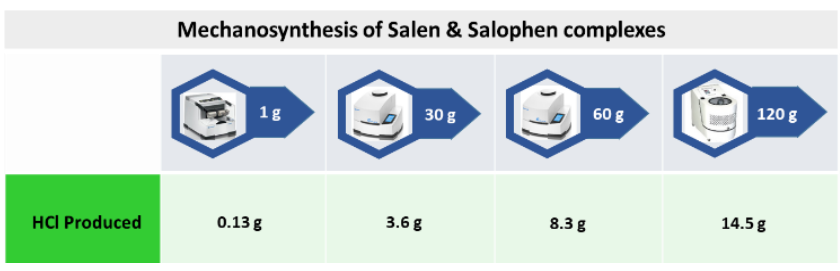

Figure 5 Amount of $\mathrm{HCl}$ produced (in grams) throughout the different mechanochemical scales

\section{Energy efficiency cost and safety considerations.}

While the environmental footprint of the process is an important parameter, other aspects such as cost, energy efficiency and safety should also be taken into consideration to determine the most suitable synthetic route. Therefore, we have also compared the energy usage, energy efficiency and cost difference between the two approaches studied. 
Table 2. Energy consumption and cost comparison.

\begin{tabular}{|c|c|c|c|c|c|c|c|c|c|}
\hline \multirow[t]{2}{*}{$\begin{array}{c}\text { Scale } \\
\text { (g) }\end{array}$} & \multicolumn{2}{|c|}{$\begin{array}{c}\text { Energy consumption } \\
\mathbf{k W} \cdot \mathbf{h} \cdot \mathrm{kg}^{-1}\left(\mathrm{MJ} \cdot \mathrm{kg}^{-1}\right)\end{array}$} & \multirow[t]{2}{*}{ Energy saved (\%) } & \multicolumn{6}{|c|}{$\begin{array}{c}\text { Approx. electricity cost } \\
\text { (USD } \cdot \mathrm{kg}^{-1} \text { ) }\end{array}$} \\
\hline & Mechanochemistry & Solution & & \multicolumn{3}{|c|}{ Mechanochemistry } & \multicolumn{3}{|c|}{ Solution } \\
\hline $0.1 \mathrm{~g}$ & $32,400(116,640)$ & $128,000(460,800)$ & 75 & 1,3576 & 5171.04 & 2099.52 & $5,363.20$ & $20,428.80$ & 8294.40 \\
\hline $1.0 \mathrm{~g}$ & $900(3240)$ & $12,800(46,080)$ & 93 & 37.71 & 143.64 & 58.32 & 536.32 & $2,042.88$ & 829.44 \\
\hline $30.0 \mathrm{~g}$ & $250(900)$ & $462.67(1,536)$ & 41 & 10.47 & 39.90 & 16.20 & 19.39 & 73.84 & 29.98 \\
\hline $120.0 \mathrm{~g}$ & $80(288)$ & $106(384)$ & 25 & 3.35 & 12.77 & 5.18 & 4.44 & 16.92 & 6.87 \\
\hline
\end{tabular}

To perform the energy consumption assessment, as a rough measure, we approximated maximum power consumption (as the stated in the PBM technical specifications) during the milling process and negligible power consumption when idle. As for the solution-based methodology, we presumed the hot plate to be at maximum power while heating and negligible power when only stirring - which is in line with previous energy consumption reports. ${ }^{62}$ Taking this as our baseline, we calculated the energy usage for both the processes based on the following equation:

Energy usage $(\mathrm{kW} \cdot \mathrm{h})=$ Power $(\mathrm{kW}) \times$ Duration $(\mathrm{h})$

(Eq. 3)

Our calculations show that the mechanochemical route required less energy on the large-scale synthesis. For synthesis of $120 \mathrm{~g}$ of $\mathbf{1}$, the PBM consumed $\sim 9.6 \mathrm{~kW} \cdot \mathrm{h}$ over 4 hours, which translated to $288 \mathrm{MJ} \cdot \mathrm{kg}^{-1}\left(80 \mathrm{~kW} \cdot \mathrm{h} \cdot \mathrm{kg}^{-1}\right)$ of product. Whereas the energy consumption for solutionbased route required $\sim 12.8 \mathrm{~kW} \cdot \mathrm{h}$ over $16 \mathrm{~h}$, which is equivalent to $384 \mathrm{MJ} \cdot \mathrm{kg}^{-1}\left(107 \mathrm{~kW} \cdot \mathrm{h} \cdot \mathrm{kg}^{-1}\right)$. The energy consumption at different scale batches is presented in Table 2. Moreover, the energy saving comparison between both methods was assessed using the following equation: ${ }^{60}$

$\%$ Energy saved $=\frac{E_{\text {sol. }}-E_{\text {mech. }}}{E_{\text {sol. }}} \times 100 \%$

Our calculations indicate that large scale solution synthesis consumed 33\% (i.e., 25\% energy savings with respect to solution-based method using Eq. 4) more energy than the mechanochemical route. In addition, while we only utilized two stations of the PBM throughout our experiments, the PBM used can hold up to 4 jars at once. Hence, based on the energy consumption assumptions used throughout our studies (vide supra), $500 \mathrm{~g}$ of product could be produced under the same energy cost (i.e., $1600 \mathrm{~kW} \cdot \mathrm{h}^{-1}$ ) provided identical reactions times are used.

Finally, to conclude our studies, we examined how energy consumption translates into industrial production cost. For this purpose, a cost range using the highest and lower average price of electricity to industrial end-user throughout the USA (excluding Pacific Noncontiguous regions - Alaska and Hawaii - owing to its hight cost), as well as the average cost in the USA was calculated (i.e., $0.0419-0.1596$ and $0.0648 \mathrm{USD}(\mathrm{KW} \cdot \mathrm{h})^{-1}$, respectively) see Table 2 and SI. ${ }^{63}$ As expected, the cost per $\mathrm{kg}$ dramatically decreases as the scale increases in all cases. In terms of the average cost per $\mathrm{kg}$ at higher scale ranges (which is most representative of industrial scales), mechanosynthetic routes are more cost-effective in all cases. It is also noted that the differences in production cost between the two methods decreases as the scale increases. However, as our calculations are solely based on energy consumption, costs associated with the use of solvents (i.e., cost, transportation, and recycling or disposal) have not been taken into consideration. Therefore, we predict that the advantage of mechanochemistry will remain even at larger scales.

\section{Conclusion}

In summary, we have demonstrated the solid-state synthesis of bromo-substituted salen and salophen ligands as well as their respective aluminium and indium complexes. Notably, the synthetic methodology explored in this study did not require the use of LAG to achieve moderate to high yields, which further highlights the environmentally benign nature of mechanosynthesis.

Using the Br-Salophen-Al complex as a model reaction, we have studied the environmental impact of both mechanochemical and solution-based synthesis by a green chemistry metrics approach. Relative to solution-based synthesis, mechanosynthesis has achieved a very low Efactor and PMI while maintaining high RME and reaction yields.

Although the $\mathrm{HCl}$ released from these reactions can go mostly unnoticed in small-scale synthesis, at larger scales, the $\mathrm{HCl}$ produced can be dangerous to handle and may lead to equipment damage. The use of triethylamine as a $\mathrm{HCl}$ scavenger can mitigate these risks, albeit at the cost of an increased E-factor and PMI. To circumvent the use of chemical bases thus maintaining the excellent green metrics and sustainability of these processes, specialised milling apparatus capable of venting excess pressure is necessary. This warrants more research on the design and engineering of improved mechanochemical milling machines for its practical implementation in the industry. 


\section{ASSOCIATED CONTENT}

Supporting Information. Experimental procedures for synthesis, detailed calculations and analytical data is provided in this file.

\section{AUTHOR INFORMATION}

\section{Corresponding Author}

* fgarcia@ntu.edu.sg.

Funding Sources

A*STAR AME IRG (A1783c0003), MOE AcRF Tier 1 (M4011709) and NTU start-up grant (M4080552)

\section{Notes}

The authors declare no competing financial interest.

\section{ACKNOWLEDGEMENTS}

$X$ F. G. would like to acknowledge A*STAR AME IRG (A1783c0003), MOE AcRF Tier 1 (M4011709) and NTU start-up grant (M4080552) for financial support. F L and V.S. would like to thank $A *$ STAR for fellowship.

\section{REFERENCES}

1. Do, J. L.; Frisčic, T. Mechanosynthesis: A force of synthesis. ACS Cent. Sci. 2017, 3, 13-19

2. Tan, D., Garcia, F. Main group mechanochemistry: from curiosity to established protocols. Chem. Soc. Rev. 2019, 48, 2274-2292.

3. Yan, X. S.; Kai, X.; Jack, C.; Ganguly, R.; Hirao, H.; Friscic, T.; García, F. Mechanochemistry Made It Possible: Synthesis of the Sterically Encumbered Phosphazane $\mathrm{P}_{4}\left(\mathrm{~N}^{t} \mathrm{Bu}\right)_{6}$. Angew. Chem. Int. Ed. 2016, $55,12736-12740$

4. Ardila-Fierro, K. J.; Bolm, C.; Hernández, J. G. Mechanosynthesis of Odd-Numbered Tetraaryl[n]cumulenes. Angew. Chem. Int. Ed. 2019, 58, 37, 12945-12949.

5. Prochowicz, D., Nawrocki, J., Terlecki, M., Marynowski, M., Lewiński, J. Facile Mechanosynthesis of the Archetypal Zn-Based Metal-Organic Frameworks. Inorg. Chem. 2018, 57, 21, 1343713442.

6. Hong, Z., Tan, D., John, R. A., Tay, Y. K. E., Ho, Y. K. T., Zhao, X., Sum, T. C., Mathews, N., García, F., Soo, H. S. Completely Solvent-free Protocols to Access Phase-Pure, Metastable Metal Halide Perovskites and Functional Photodetectors from the Precursor Salts. iScience. 2019, 16, 312-325.

7. Hernández, J. G., Bolm, C. Altering Product Selectivity by Mechanochemistry. J. Org. Chem. 2017, 82, 8, 4007-4019

8. Ardila-Fierro, K. J.; Crawford, D. E.; Körner, A.; James, S. L.; Bolm, C.; Hernández, J. G. Papain-catalysed mechanochemical synthesis of oligopeptides by milling and twin-screw extrusion: application in the
Juliá-Colonna enantioselectiveepoxidation. Green Chem. 2018, 20 1262-1269.

9. Shi, X. Y., Martin, K. A., Liang, R. Z., Starr, D. G., Li, Y., Ganguly, R., Díaz, J., García, F. Synthesis of Unique Phosphazane Macrocyclices via Steric Bond Activation. Inorg. Chem. 2018, 57, 10993-11004

10. Sim, Y., Tan, D., Ganguly, R., Li, Y., García, F. Orthogonality in Main Group Compounds: Direct One-step Synthesis of Air- and Moisture-stable Cyclophosphazanes by Mechanochemistry Chem. Comm. 2018, 54, 6800-6803.

11. Friščić, T.; Mottillo, C.; Titi, H. M. Mechanochemistry for Synthesis. Angew. Chem. Int. Ed. 2020, 59, 3, 1018-1829.

12. Hernández, J. G.; Butler, I. S.; Friščić, T. Multi-step and multicomponent organometallic synthesis in one pot using orthogonal mechanochemical reactions. Chem. Sci. 2014, 5, 3576-3582.

13. Hernández, J. G.; Macdonald, N. A. J.; Mottillo, C.; Butler, I. S.; Friščić, T. A mechanochemical strategy for oxidative addition: remarkable yields and stereoselectivity in the halogenation of organometallic $\operatorname{Re}(\mathrm{I})$ complexes. Green Chem. 2014, 16, 10871092.

14. Shaw, T. E.; Mathivathanan, L.; Jurca, T. One-Pot, One-Step Precatalysts through Mechanochemistry. Organometallics 2019, 38, 21, 4066-4070.

15. Ferguson, M.; Giri, N.; Huang, X.; Apperley, D.; James, S. L. Onepot two-step mechanochemical synthesis: ligand and complex preparation without isolating intermediates. Green Chem. 2014, 16, 1374-1382.

16. Batzdorf L.; Zientek N.; Rump D.; Fischer F.; Maiwald M.; Emmerling F. Make and break - Facile synthesis of cocrystals and comprehensive dissolution studies. J. Mol. Struct. 2017, 1133, 1823.

17. Fischer F.; Heidrich A.; Greiser S.; Benemann S.; Rademann K.; Emmerling F. Polymorphism of mechanochemically synthesized cocrystals: A case study. Cryst. Growth Des. 2016, 16, 1701-1707.

18. Oh, S. Y.; Nickels, C. W.; García, F.; Jones, W.; Friščić, T. Switching between halogen- and hydrogen-bonding in stoichiometric variations of a co-crystal of a phosphine oxide. Cryst. Eng. Comm 2012, 14, 6110 - 6114 .

19. Huang Y. H.; Lo W. S.; Kuo Y. W.; Chen W. J.; Lin C. H.; Shieh F. K. Green and rapid synthesis of zirconium metalorganic frameworks via mechanochemistry: Ui0-66 analog nanocrystals obtained in one hundred seconds. Chem. Commun. 2017, 53, 5818-5821.

20. Matoga D.; Oszajca M.; Molenda M. Ground to conduct: mechanochemical synthesis of a metal-organic framework with high proton conductivity. Chem. Commun. 2015, 51, 7637-7640.

21. Atkinson M. B. J.; Bucar D. K.; Sokolov A. N.; Friscic T.; Robinson C. N.; Bilal M. Y.; Sinada N. G.; Chevannes A.; MacGillivray L. R. General application of mechanochemistry to templated solid-state reactivity: rapid and solvent-free access to crystalline supermolecules. Chem. Commun. 2008, 5713-5715.

22. Pascu M.; Ruggi A.; Scopelliti R.; Severin K. Synthesis of borasiloxane-based macrocycles by multicomponent condensation reactions in solution or in a ball mill. Chem. Commun. 2013, 49, 4547. 
23. Mi G.; Saito F.; Hanada M. Mechanochemical synthesis of tobermorite by wet grinding in a planetary ball mill. Powder Technol. 1997, 93, 77-81.

24. Zhong L. H.; Qu J.; Li X. W.; He X. M.; Zhang Q. W. Simultaneous synthesis of ettringite and absorbate incorporation by aqueous agitation of a mechanochemically prepared precursor. RSC Adv. 2016, 6, 35203-35209.

25. Jacobsen, E. N.; Zhang, W.; Muci, A. R.; Ecker, J. R.; Deng, L. J. Am. Chem. Soc. 1991, 113, 7063-7064.

26. Gualandi, A.; Marchini, M.; Mengozzi, L.; Kidanu, H. T.; Franc, A.; Ceroni, P.; Cozzi, P. G.. Aluminum(III) Salen Complexes as Active Photoredox Catalysts. Eur. J. O. C, 2020, 10, 1486-1490.

\section{Cozzi, P. G. Chem. Soc. Rev. 2004, 33, 410-421.}

28. Van Zee, N. J.; Sanford, M. J.; Coates, G. W. J. Am. Chem. Soc. 2016 , $138,2755-2761$

29. Stober, T.; Mulryan, D.; Williams, C. Switch Catalysis To Deliver Multi-Block Polyesters from Mixtures of Propene Oxide, Lactide, and Phthalic Anhydride. Angew. Chem. Int. Ed. 2018, 57, 1689316897.

30. Specklin, D.; Fliedel, C.; Hild, F.; Mameri, S.; Karmazin, L.; Bailly, C.; Dagorne, S. Mononuclear Salen-Gallium Complexes for Isoselective Ring-Opening Polymerization (ROP) of rac-Lactide. Dalton Trans. 2017, 46, 12824.

31. Germain, M. E.; Knapp, M. J. Inorganic Chemistry 2008, 47, 9748- 9750

32. Xu, Y.; Meng, J.; Meng, L.; Dong, Y.; Cheng, Y.; Zhu, C. Chemistry A European Journal 2010, 16, 12898-12903.

33. Bhattacharjee, C. R.; Das, G.; Mondal, P. European Journal of Inorganic Chemistry 2011, 5390-5396.

34. Vashchenko, A. A.; Lepnev, L. S.; Vitukhnovskii, A. G.; Kotova, O. V.; Eliseeva, S. V.; Kuz'mina, N. P. Optics and Spectroscopy 2010, $108,463-465$.

35. Liang, Y.; Duan, R-L.; Hu, C-Y.; Pang, X.; Zhang, W-X.; Chen, X-S. Salen-iron Complex: Synthesis, Characterisation and Their Reactivity With Lactide. Chinese Journal of Polymer Science 2018 , 36, 185-189.

36. Uysal, S.; Koc, Z. E.; The Synthesis and Characterization of (MSalen/Salophen/saldeta/salpy) [M= Fe(III) or $\mathrm{Cr}(\mathrm{III})]$ Capped Heteronuclear Schiff Bases-Dioxime Ni(II) Complexes: Their Thermal and Magnetic Behaviours. Journal of Molecular Structure 2018, 1165, 14-22.

37. Cheng, J.; Wei, K.; Ma, X.; Zhou, X.; Xiang, H. Synthesis and Photophysical Properties of Colourful Salen-Type Schiff Bases. J. Phys. Chem.C 2013, 117, 32, 16552-16563.

38. Zhang, X.; Shi, J.; Song, J.; Wang, M.; Xu, X.; Qu, L.; Zhou, X.; Xiang, H. Non-conmjugated Fluorescent Molecular Cages of Trinuclear Fluoroborate Complexes with Salicyaldehyde-Based Schiff Base Ligands. ACS Omega 2018, 3, 8992-9002.

39. Raj, P.; Singh, A,; Singh, A.; Singh, N. Synthesis and Photophysical Properties of Schiff Bases Ni(II) Complexes:
Application for Sustainable Antibacterial Activity and Cytotoxicity. ACS Sustainable Chemistry \& Engineering 2017, 5 (7), 6070-6080.

40. Johnstone, N. C.; Azam, E. S.; Hitchcock, P. B.; Fulton, J. R. Synthesis of Aluminium Complexes Bearing a Piperazine-Based Ligand System. J. Organomet. Chem. 2010, 695, 170-176.

41. Sheldon, R. A. Fundamentals of green chemistry: efficiency in reaction design. Chem. Soc. Rev. 2012, 41, 1437-1451.

42. Roschangar, F.; Sheldon, R. A.; Senanayake, C. H. Overcoming barriers to green chemistry in the pharmaceutical industry - the Green Aspiration Level concept. Green Chem. 2015, 17 (2), 752-768.

43. Qureshi, Z. S.; Deshmukh, K. M.; Bhanage, B. M. Applications of ionic liquids in organic synthesis and catalysis. Clean Technol. Environ. Policy 2014, 16, 1487-1513

44. Sheldon, R. A. Green solvents for sustainable organic synthesis: state of the art. Green Chem. 2005, 7, 267-278.

45. Frontmatter. In Green Reaction Media in Organic Synthesis; Blackwell Publishing Ltd, 2007; pp i-xi

46. Lively, R. P.; Sholl, D. S. From Water to Organics in Membrane Separations. Nat. Mater. 2017, 16, 276-279.

47. Leoni, L.; Carletta, A.; Fusaro, L.; Dubois, J.; Tumanov, N. A.; Aprile, C.; Wouters, J.; Cort, A. D. A Simple and Efficient Mechanochemical Route for the Synthesis of Salophen Ligands and of the Corresponding Zn, Ni, and Pd Complexes. Molecules 2019, $24,2314$.

48. Milbeo, P.; Quintin, F.; Moulat, L.; Didierjean, C.; Martinez, J.; Bantreil, X.; Calmes, M.; Lamaty, F. Synthesis, Characterization and Cytotoxic Activity Evaluation of New Metasalen Complexes Based on the 1,2-bicyclo[2.2.2] octane Bridge. ChemRxiv 2020.

49. Seo, T.; Ishiyama, T.; Kubota, K.; Ito, H. Solid state SuzukiMiyaura cross-coupling reactions: olefin-accelerated C-C coupling using mechanochemistry. Chem. Sci. 2019, 10, 8202-8210.

50. Kubota, K.; Seo, T.; Koide, K.; Hasegawa, Y.; Ito, H. Olefin accelerated solid state $\mathrm{C}-\mathrm{N}$ cross coupling reactions using mechanochemistry. Nat. Comm. 2019, 10, 111.

51. Vigato, P.A.; Tamburini, S. The challenge of cyclic and acyclic Schiff bases and related derivatives. Coord. Chem. Rev. 2004, 248, $1717-2128$

52. M. D. M. C. Ribeiro da Silva, J. M. Gonçalves, A. L. R. Silva, P. C. F. C. Oliveira, B. Schröder and M. A. V. Ribeiro da Silva, Journal of Molecular Catalysis A: Chemical 2004, 224, 207-212.

53. Jiang, Z. J.; Li, Z-H.; Yu, J-B.; Su, W.-K. Liquid-Assisted Grinding Accelerating: Suzuki-Miyaura Reaction of Aryl Chlorides under High-Speed Ball-Milling Conditions. J. Org. Chem. 2016, 81, 20, 10049-10055.

54. Stolle, A.; Schmidt, R.; Jacob, K. Scale-up of organic reactions in ball mills: process intensification with regard to energy efficiency and economy of scale. Faraday Discuss. 2014, 170, 267- 286.

55. Baláž, P. Mechanochemistry in Nanoscience and Minerals Engineering; Springer-Verlag: Berlin, 2008. 
56. Burmeister, C. F.; Kwade, A. Process engineering with planetary ball mills. Chem. Soc. Rev. 2013, 42, 7660- 7667

57. Lapkin, A.; Constable, D. J. C. Green Chemistry Metrics: Measuring and Monitoring Sustainable Processes. 2008, Print ISBN: 9781405159685 |Online ISBN:9781444305432

58. Sheldon, E. The E-factor: fifteen years on. Green Chem. 2007, 9, 1273-1283.

59. Manley, J. B.; Kopach, M. E.. Greener Pharmaceutical Science Through Collaboration: The ACS GCI Pharmaceutical Roundtable. Wiley, 2018, Chapter 24, 649-674.

60. Jimenez-Gonzalez, C.; Ponder, C. S.; Broxterman, Q. B.; Manley, J. B. Using the Right Green Yardstick: Why Process Mass Intensity Is Used in the Pharmaceutical Industry To Drive More Sustainable Processes. Org. Proc. Res. Dev. 2011, 15, 912-917.

61. Andraos, J. Relationships between step and cumulative PMI and E-factors: implications on estimating material efficiency with respect to charting synthesis optimization strategies. Green Process Synth. 2019, 8, 324-336.

62. Chopra, J.; Dineshkumar, R.; Bhaumik, M.; Dhanarajan, G.; Kumar, R. R.; Sen, R. Integrated in situ transesterification for improved biodiesel production from oleaginous yeast: a value proposition for possible industrial implication. $R S C A d v$. 2016, 6, 70364-70373.

63.www.eia.gov/electricity/monthly/epm_table_grapher.php?t=e pmt_5_6_a 